\title{
Corneal epithelial disorders associated with the anticancer drug S-1
}

\author{
Hiroshi Osawa • Takeshi Hashimoto • \\ Hiroaki Goto • Tomohiro Myojo • Yoshinobu Mizuno
}

Received: 15 October 2012/ Accepted: 8 January 2013/Published online: 2 February 2013

(C) The Japan Society of Clinical Oncology 2013

\begin{abstract}
We present the cases of three patients with colorectal cancer who were treated with combined chemotherapy regimens containing S-1. Eye disorders developing as adverse effects of anticancer drugs can be resolved by discontinuation of the causative anticancer drugs, although the disorders may be irreversible in some cases, thereby necessitating early diagnosis and prompt treatment. Medical oncologists must pay attention to eye disorders caused by the anticancer drug S-1. It is necessary to refer patients with eye disorders suspected to be caused by an anticancer drug immediately to an ophthalmologist, because of the possibility of injury to the cornea and/or lacrimal duct.
\end{abstract}

Keywords S-1 C Corneal epithelial disorders

\section{Introduction}

S-1 is a key oral anticancer drug used in the treatment of gastrointestinal cancer [1-3]; the drug consists of a mixture of a prodrug of 5-fluorouracil (5-FU), namely tegafur (FT), and two modulators, viz. 5-chloro-2,4-dihydroxypyridine (CDHP) and potassium oxonate (Oxo). S-1 exerts high antitumor effects and elicits fewer adverse events, especially fewer gastrointestinal adverse events, than intravenous 5-FU. On the other hand, Esmaeli et al. [4] reported

H. Osawa $(\bowtie) \cdot$ T. Hashimoto $\cdot$ H. Goto · T. Myojo

Department of Oncology and Hematology, Edogawa Hospital,

2-24-18 Higashi-koiwa, Edogawa, Tokyo 133-0052, Japan

e-mail: oosawa@edogawa.or.jp

Y. Mizuno

Department of Ophthalmology, Edogawa Hospital, Tokyo, Japan the occurrence of severe canalicular stenosis and nasolacrimal duct blockage after S-1 therapy. Ito et al. [5] reported the development of corneal epithelial lesions following treatment with S-1. The SPIRITS trial also reported the occurrence of eye disorders (epiphora) at a comparatively high rate $(17.6 \%)$ in patients receiving treatment with $\mathrm{S}-1$. Thus, medical oncologists must begin to pay attention to these eye disorders associated with S-1, including epiphora, keratitis, eye pain, etc. We present two cases of corneal epithelial disorders that developed after the start of treatment with the anticancer drug S-1, to emphasize the need for regular follow-up of such patients to avoid irreversible visual loss.

\section{Case report}

\section{Case 1}

A 62-year-old man who was admitted to our hospital with the chief complaint of melena was diagnosed as having rectal cancer (Table 1). Miles operation was performed on 1 July 2009, and the pathological findings were as follows: Locus $\mathrm{Rb}$, type $2,4.5 \times 5 \mathrm{~cm}$, tub1, pA, ly1, v3, N1 (1/47), pStageIIIA. Adjuvant UFT/L-leucovorin (LV) chemotherapy was administered from 5 August 2009 to 2 February 2010. Computed tomographic follow-up revealed multiple lung metastases and a solitary liver metastasis 6 months after completion of the adjuvant chemotherapy. First-line Bmab + SOX chemotherapy was initiated [bevacizumab (Bmab): $7.5 \mathrm{mg} / \mathrm{kg}$ on day $1,1 \mathrm{~h}$ intravenous infusion (iv); oxaliplatin (L-OHP): $130 \mathrm{mg} / \mathrm{m}^{2}$ on day 1 , 2-h iv, $\mathrm{S}-1$ : $80 \mathrm{mg} / \mathrm{m}^{2}$ from days 1 to 15 , orally]. Re-evaluation by CT after 15 courses of chemotherapy revealed progression of the lung metastatic lesions. 
Table 1 Patient characteristics

\begin{tabular}{llll}
\hline & Patient 1 & Patient 2 & Patient 3 \\
\hline Age, gender & $62, \mathrm{M}$ & $69, \mathrm{M}$ & $63, \mathrm{~F}$ \\
ECOG PS & 0 & 0 & 1 \\
Primary & Rectum & Ascending colon & Sigmoid colon \\
Chemotherapy regimen & Bevacizumab + IRIS & SOX & Bevacizumab + IRIS \\
Eye disorders & Epiphora & Eye pain & Eye pain \\
Keratopathy pattern & Superficial vortex & Superficial vortex & Superficial vortex \\
Discontinuation period of S-1 & 3 months & 8 weeks & 6 weeks \\
Corneal epithelial disorders & Improved & Improved & Improved \\
\hline
\end{tabular}

$M$ male, $F$ female, ECOG PS Eastern Cooperative Oncology Group performance status, IRIS CPT-11 + S-1, SOX oxaliplatin + S-1

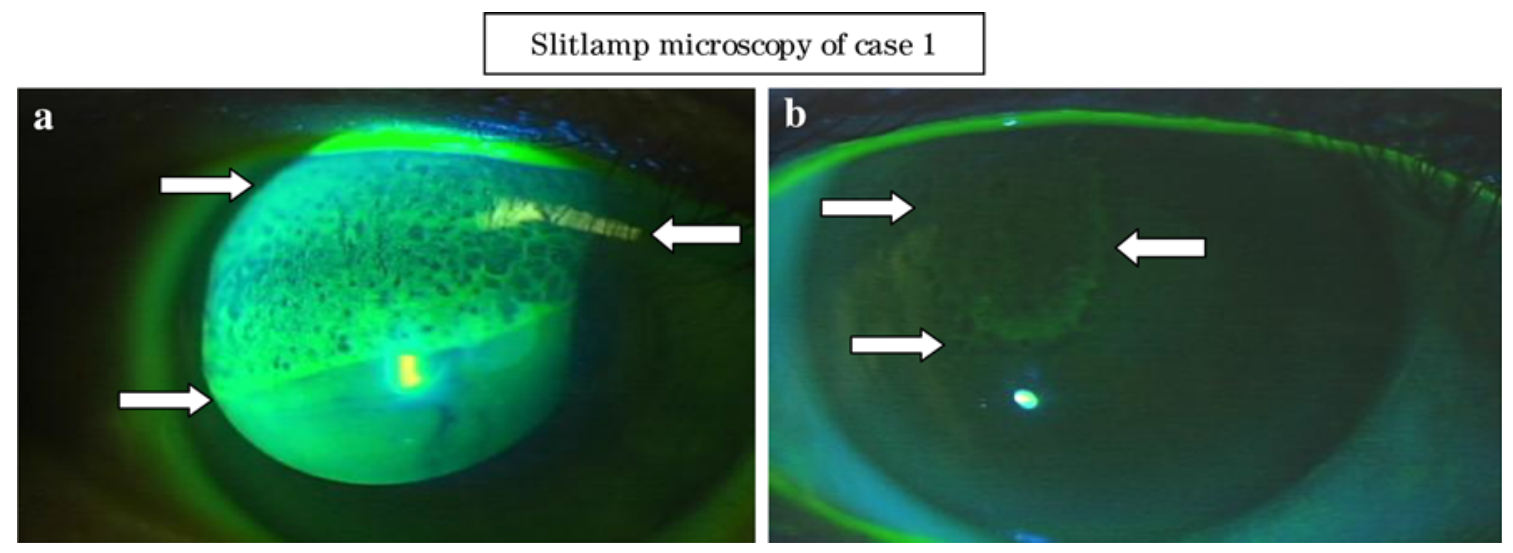

Fig. 1 a Superficial vortex-pattern keratopathy (white arrows) on the upper areas of right eye. b Improving superficial vortex-pattern keratopathy (white arrows) on the upper areas of right eye 3 months later without S-1

Therefore, the patient was started on second-line Bmab + IRIS chemotherapy (Bmab: $7.5 \mathrm{mg} / \mathrm{kg}$ on day $1,1-\mathrm{h}$ iv; CPT-11: $150 \mathrm{mg} / \mathrm{m}^{2}$ on day 1, 1.5 -h iv; S-1: $80 \mathrm{mg} / \mathrm{m}^{2}$ from days 1 to 15 , orally) from 14 November 2011. After 5 courses of the second-line chemotherapy, the patient presented with epiphora. He was diagnosed as having keratitis caused by S-1 on 6 June 2012 at our department of ophthalmology. S-1 was immediately discontinued, and from the 7th course of chemotherapy onward, the patient received chemotherapy without S-1 (Bmab + CPT-11). Although this resulted in improvement of the corneal epithelial disorder, the patient continues to suffer from residual visual disorder. On ophthalmological examination, the patient's best corrected visual acuity was 20/60 in the right eye and 20/25 in the left eye. Slit-lamp examination showed superficial punctate keratitis in the upper area in the right eye, and hurricane keratitis in the upper and temporal areas of the left eye. The hurricane keratitis has been improving without S-1 chemotherapy gradually (Fig. 1a).

\section{Case 2}

A 69-year-old man who was admitted to our hospital complaining of abdominal fullness was diagnosed as having ascending colon cancer (Table 1). Right proctocolectomy was performed on 1 June 2012. The pathological findings were as follows: Locus A, type $2,3.3 \times 3 \mathrm{~cm}$, tub1-2, pSE, ly1, v1, N1 (1/29), pStageIIIA. Adjuvant SOX chemotherapy (oxaliplatin: $130 \mathrm{mg} / \mathrm{m}^{2}$ on day $1,2-\mathrm{h}$ iv; $\mathrm{S}-1: 80 \mathrm{mg} / \mathrm{m}^{2}$ on days $1-15$, orally) was started 5 weeks after the surgery. During the second course of chemotherapy, on 4 August 2012, the patient complained of eye pain and dyspnea and was immediately admitted to our hospital. He was diagnosed as having keratitis caused by S-1 on 6 August 2012 at our department of ophthalmology. SOX therapy was discontinued immediately, and the corneal epithelial disorder has not progressed since. Ophthalmological examination revealed the following findings: the best corrected visual acuity was $20 / 20$ in the right eye and not beyond hand motion in the left eye. Slit lamp examination showed hurricane keratitis in the upper temporal areas and mature cataract in both eyes (Fig. 2a).

\section{Case 3}

A 63-year-old woman who was admitted to our hospital with perforation of the colon and was diagnosed as having sigmoid colon cancer (Table 1). Light proctocolectomy 


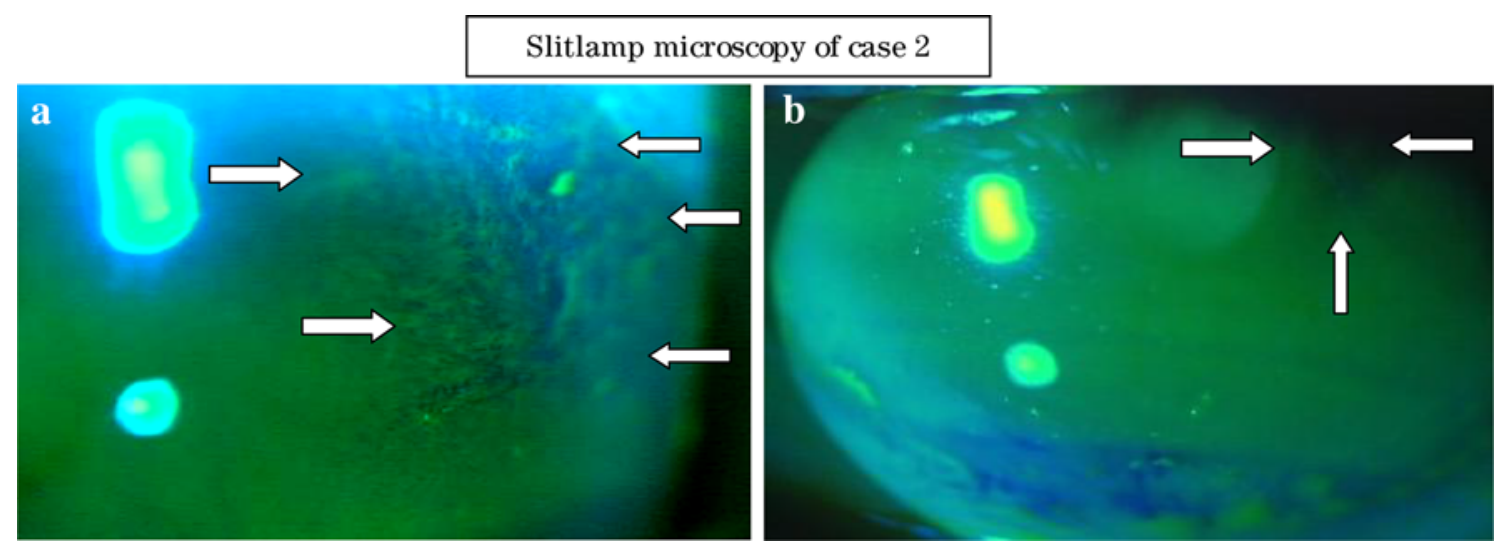

Fig. 2 a Photo of case 2; vortex-pattern keratopathy (white arrows) on the upper and temporal areas and mature cataract of right eye. b Improving superficial vortex-pattern keratopathy (white arrows) on the upper areas of right eye 8 weeks later without S-1

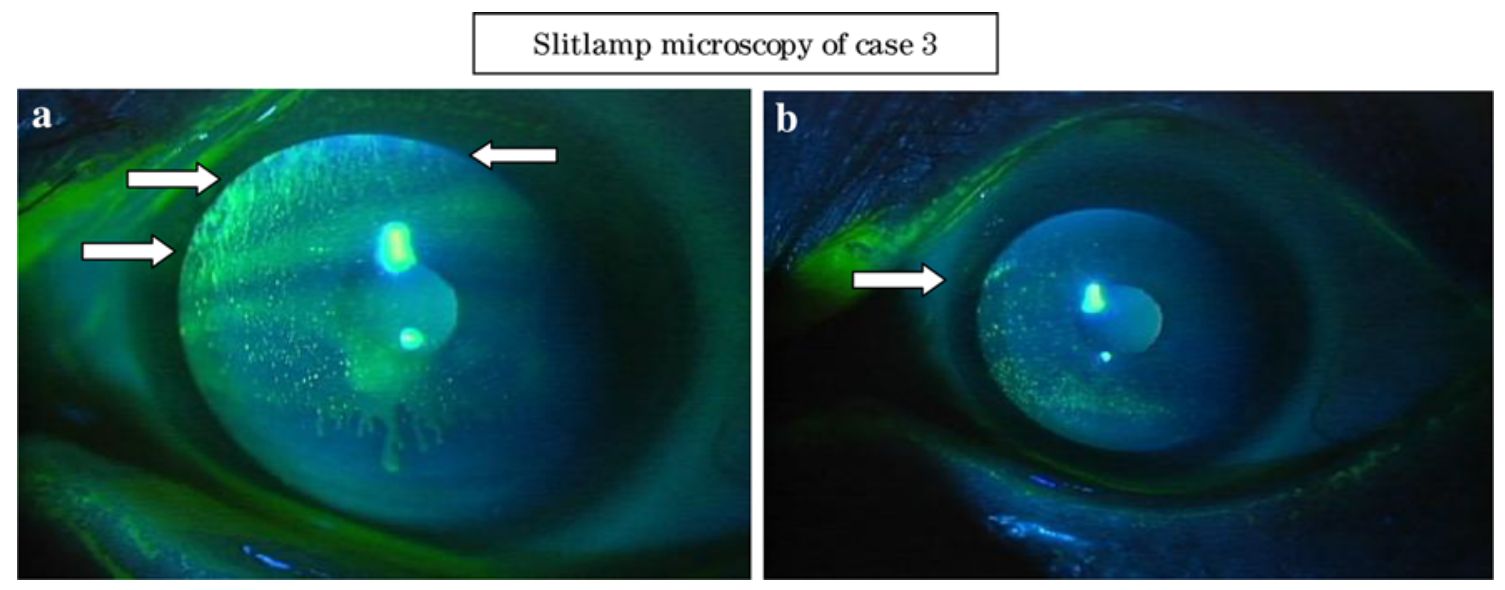

Fig. 3 a Photo of case 2; vortex-pattern keratopathy (white arrows) on the upper and temporal areas and mature cataract of right eye. b Improved superficial vortex-pattern keratopathy with a crack line

and right liver lobectomy were performed on 27 April 2007, and the pathological findings were as follows: Locus Rs, type 2, $30 \times 40 \mathrm{~cm}$, tub1, pSS, ly2, v0, N0 (0/28), H3, pStageIV. Adjuvant setting FOLFOX4 (L-OHP, 5-FU, LV) chemotherapy was administered from 11 June 2007 to 18 September 2007. Computed tomographic follow-up revealed multiple lung metastases 6 months after completion of the FOLFOX4. First-line chemotherapy was initiated by 34 courses of Bmab + mFOLFOX6 (Bmab: $5 \mathrm{mg} / \mathrm{kg}$ on day 1, 1-h iv; L-OHP: $85 \mathrm{mg} / \mathrm{m}^{2}$ on day 1 , 2-h iv; iv 5-FU: $400 \mathrm{mg} / \mathrm{m}^{2}$ on day 1 ; continuous iv (civ) 5 -FU: $2400 \mathrm{mg} / \mathrm{m}^{2}$ on day $\left.1-2\right)$. Second-line chemotherapy was initiated by 11 courses of Bmab + FOLFIRI (Bmab: $5 \mathrm{mg} / \mathrm{kg}$ on day $1,1-\mathrm{h}$ iv; CPT-11: $150 \mathrm{mg} / \mathrm{m}^{2}$ on day $1,1.5$-h iv; iv 5-FU: $400 \mathrm{mg} / \mathrm{m}^{2}$ on day 1 ; civ 5 -FU: $2400 \mathrm{mg} / \mathrm{m}^{2}$ on day $1-2$ ) because of the lung metastatic lesions. Actually, if her v-Ki-ras2 Kirsten rat sarcoma viral oncogene homolog (KRAS) were wild-type, we should administer chemotherapy with anti-epidermal growth factor receptor (EGFR) remains (white arrow) on the upper areas of right eye 6 weeks later without S-1

drugs (i.e., cetuximab, panitumumab). Unfortunately, her KRAS was mutated (codonG12A). Therefore, the patient was started on third-line chemotherapy (Bmab: $7.5 \mathrm{mg} / \mathrm{kg}$ on day 1, 1-h iv; CPT-11: $150 \mathrm{mg} / \mathrm{m}^{2}$ on day $1,1.5$-h iv; S-1: $80 \mathrm{mg} / \mathrm{m}^{2}$ from days 1 to 15 , orally). After 2 courses of the third-line chemotherapy, the patient presented with epiphora. He was diagnosed as having keratitis caused by S-1 on 26 August 2012 at our department of ophthalmology. S-1 was immediately discontinued, and from the $3 \mathrm{rd}$ course of chemotherapy onward, the patient received chemotherapy without S-1 (Bmab + CPT-11). Although this resulted in improvement of the corneal epithelial disorder, the patient continues to suffer from residual visual disorder. On ophthalmological examination, the patient's best corrected visual acuity was 20/40 in the right eye and 20/25 in the left eye. Slit-lamp examination showed superficial punctate keratitis in the upper area in the right eye, and hurricane keratitis in the upper and temporal areas of the left eye (Fig. 3a). 


\section{Discussion}

In Japan, S-1, an oral anticancer drug, is frequently used in adjuvant chemotherapy for advanced gastric cancer (ACTS-GC [1]), systemic chemotherapy for advanced and recurrent gastric cancer (SPIRITS trial [2]), non-small cell lung cancer (LETS trial [3]), pancreatic cancer (GEST trial [6]), etc. S-1 is composed of a mixture of FT, a prodrug of 5-FU, CDHP, and Oxo. CDHP reversibly inhibits degradation of 5-FU by dihydropyrimidine dehydrogenase (DPD), resulting in sustained high serum concentrations of 5-FU, thereby enhancing the antitumor activity of FT. Oxo is selectively distributed to the gastrointestinal tract, where the drug in high concentrations inhibits orotate phosphoribosyltransferase, which phosphorylates 5-FU to its active metabolite in humans. Inhibition of this enzyme reduces the gastrointestinal toxicity of 5-FU [7]. The cytocidal mechanism of 5-FU is roughly divided into inhibition of DNA synthesis and RNA dysfunction. The former is known to depend on the duration of exposure to 5-FU, whereas the latter depends on the concentration of 5-FU [8]. It is possible to achieve sustained high concentrations of 5-FU in patients with S-1, although adverse effects are still induced in sensitive organs and tissues [7,9]. Bone marrow suppression, skin rash, gastrointestinal toxicities (anorexia, nausea, vomiting), and fatigue have been reported as the major adverse effects of S-1 therapy [7, 10], with the alimentary tract mucosa and skin, both of which are multilayered epithelia consisting of cells with high metabolic turnover rates, being especially sensitive to the drug toxicity $[11,12]$.

Corneal epithelial disorder associated with S-1 was reported by Esmaeli et al. [4] in 2005. Ito and Tanaka [5] were the first in Japan to report a case of corneal epithelial disorder caused by S-1.

Kurihara et al. [12] reported that there are two kinds of drug-induced corneal epithelial disorders, namely sheetlike lesions and superficial punctate keratopathy (SPK)-like lesions. The ophthalmologist diagnosed SPK-like lesions caused by S-1 in three cases in this study. Several solid cancers (gastric, colon, lung, breast, etc.) are treated with S-1 or capecitabine. The reported incidence rate of S-1induced corneal epithelial disorders is $2.8 \%$ (3/108). On the other hand, the estimated incidence rate of capecitabine-induced corneal epithelial disorders in 2012 was zero (total 87 cases).

However, the potential of combined S-1 or capecitabine therapy to cause corneal epithelial disorders could be overlooked, and caution is necessary to detect eye manifestations in patients receiving this therapy. In addition, it has been reported that ocular toxicity frequently involves rapidly dividing corneal epithelial cells, corneal epithelial stem cells, and lacrimal duct epithelial cells, like in the patients reported in this study. However, the symptoms and timing of onset of ocular toxicity are variable. Symptoms include eye pain and visual disturbance in corneal disorders, and metamorphopsia (distorted images) and micropsia (small images) in retinal disorders. Tachibana et al. [13] classified corneal epithelial disorders associated with TS-1 into 4 groups, type I to IV, and assessed the timing of onset and characteristics of the toxicities in these groups: type I, within 3 months after the start of treatment with TS-1, Thygeson's superficial punctate keratitis-like punctate lesions dispersed over the whole cornea; type II, 3 months to 1 year after the start of treatment with TS-1, mainly hurricane-type superficial punctate keratopathy with an epithelial crack line; type III, 3 months to 1 year after the start of treatment with TS-1, white elevated lesions; type IV, 3 months to 1 year after the start of treatment with TS-1, mainly semicircular to circular lesions based on the corneal limbus. According to this classification, case 1 in this study was categorized as type II, because the clinical symptom of metamorphopsia (distorted images) developed approximately 1 year after the initiation of TS- 1 treatment and hurricane-type superficial punctate keratopathy with an epithelial crack line was mainly observed by slit-lamp microscopy. Case 2 and 3 were also categorized as type II, because the patient developed the clinical symptom of eye pain within 3 months of the start of TS- 1 treatment and hurricane-type superficial punctate keratopathy was mainly observed by slit-lamp microscopy. In addition, Tachibana et al. [13] described that continuation of S-1 in patients with corneal disorders may lead to irreversible corneal changes.

In regard to the treatment, the ophthalmologist recommended discontinuation of S-1 and prescribed diquafosol sodium and fluorometholone eye drops. When we discontinue S-1, these eye disorders improve gradually (Figs. 1b, $2 b, 3 b)$.

Since case 2 had undergone surgery, it was possible to postpone or even discontinue the adjuvant chemotherapy. On the other hand, in case 1 and 3, it was impossible to stop systemic chemotherapy, because of the risk of recurrence of rectal cancer. We discussed change to another oral anticancer drug, capecitabine. Capecitabine is metabolized in a three-step process to the active agent, 5-FU, a known ocular irritant $[14,15]$. Although ocular irritation has been reported in $10 \%$ of patients receiving capecitabine, it is difficult to change between oral and infusional FU. Actually, the rate of incidence of eye disorders associated with S-1 treatment is higher than that associated with capecitabine treatment. Why do eye disorders occur at a higher frequency with S-1 treatment? We guess that the reason will be the structures of the anticancer agents. S-1 contains CDHP, which reversibly inhibits degradation of 5-FU by DPD, resulting in sustained high serum concentrations of 
5-FU in the blood. Sustained high concentrations of the drug are associated with an elevated risk of development of corneal epithelial disorders. We therefore avoided using FU drugs in our patients; discontinuation of FU-based drug is recommended in similar cases. Alternatively, another anticancer drug caused the eye disorders. Johnson et al. [16] reported that persistent corneal epithelial defect is associated with erlotinib. Maculopathy is associated with docetaxel [17, 18], paclitaxel [19-21], and tamoxifen [22, 23]. Optic nerve disorder is associated with 5-FU [24], paclitaxel [25], and tamoxifen [26, 27]. In the attached document information, each drug causing impairment of the visual acuity is listed, less than visual impairment $5 \%$ or frequency ignorance together.

At present, oral FU drugs (S-1 and capecitabine) are key drugs used in the treatment of gastrointestinal cancer. Medical oncologists are often called upon to treat advanced and recurrent cancers. The onset of eye disorders in particular can deteriorate the quality of life of cancer patients. Therefore, attention must be paid to the possible development of eye disorders during treatment with $\mathrm{S}-1$ or other anticancer drugs. It is necessary to refer such patients with eye disorders promptly to an ophthalmologist and postpone anticancer drug S-1, because of the possibility of injury to the cornea and/or lacrimal duct.

Conflict of interest None of the authors of this manuscript have any conflicts of interests to declare.

\section{References}

1. Sakuramoto S, Sasako M, Yamaguchi T et al (2007) Adjuvant chemotherapy for gastric cancer with S-1, an oral fluoropyrimidine. N Engl J Med 357(18):1810-1820

2. Koizumi W, Narahara H, Hara T et al (2008) S-1 plus cisplatin versus S-1 alone for first-line treatment of advanced gastric cancer (SPIRITS trial): a phase III trial. Lancet Oncol 9:215-221

3. Hirashima T, Okamoto I, Yoshioka H et al (2011) Phase III study comparing the effects of carboplatin plus S-1 and carboplatin plus paclitaxel in chemotherapy-naive patients with advanced nonsmall cell lung cancer: an updated report of the LETS study (WJTOG3605). J Clin Oncol 29(suppl): abstract 7552

4. Esmaeli B, Golio D, Lubecki L et al (2005) Canalicular and nasolacrimal duct blockage: an ocular side effect associated with the antineoplastic drug S-1. Am J Ophthalmol 140(2):325-327

5. Ito S, Tanaka A (2006) Three cases of corneal disorders associated with an oral anticancer drug S-1. Nippon Ganka Gakkai Zasshi 110:919-923

6. Ioka T, Ikeda M, Ohkawa H et al (2011) Randomized phase III study of gemcitabine plus S-1 (GS) versus S-1 versus gemcitabine (GEM) in unresectable advanced pancreatic cancer $(\mathrm{PC})$ in
Japan and Taiwan: GEST study. J Clin Oncol 29(suppl): abstract 4007

7. Shirasaka T, Shimamato Y, Ohshimo H et al (1996) Development of a novel form of an oral 5-fluorouracil derivative (S-1) directed to the potentiation of the tumor selective cytotoxicity of 5-fluorouracil by two biochemical modulators. Anticancer Drugs 7:548-557

8. Sobrero AF, Aschele C, Guglielmi AP et al (1993) Synergism and lack of cross-resistance between short-term and continuous exposure to fluorouracil in human colon adenocarcinoma cells. J Natl Cancer Inst 85:1937-1944

9. Toide H, Akiyoshi H, Minato Y et al (1977) Comparative studies on the metabolism of 2-(tetrahydrofuryl)-5-fluorouracil and 5-fluorouracil. Gann 68:553-560

10. Maehara Y (2003) S-1 in gastric cancer: a comprehensive review. Gastric Cancer 6(Suppl 1):2-8

11. Logan RM, Stringer AM, Bowen JM et al (2007) The role of proinflammatory cytokines in cancer treatment-induced alimentary tract mucositis: pathobiology, animal models and cytotoxic drugs. Cancer Treat Rev 33:448-460

12. Kurihara Y, Hiraoka T, Sakata N (2007) Corneal epithelial lesion presumably due to anticancer drug TS-1. Rinsho Ganka 61(6):969-973

13. Tachibana A, Inada N, Shoji J et al (2009) Clinical study of corneal epithelial lesion associated with anticancer drug TS $-1^{\circledR}$. Ganka 51(6):791-797

14. Dooley M, Goa KL (1999) Capecitabine. Drugs 58:69-76

15. Fraunfelder FW, Fraunfelder FT (2004) Adverse ocular drug reactions recently identified by the National Registry of DrugInduced Ocular Side Effects. Ophthalmology 111(7):1275-1279

16. Johnson KS, Levin F, Chu DS (2009) Persistent corneal epithelial defect associated with erlotinib treatment. Cornea 28:706-707

17. Teitelbaum BA, Tresley DJ (2003) Cystic maculopathy with normal capillary permeability secondary to docetaxel. Optom Vis Sci 80:277-279

18. Telander DG, Sarrraf D (2007) Cystic macular edema with docetaxel chemotherapy and the fluid retention syndrome. Semin Ophthalmol 22:151-153

19. Joshi MM, Garretson BR (2007) Paclitaxel maculopathy. Arch Ophthalmol 125:709-710

20. Smith SV, Benz MS, Brown DM (2008) Cystoid macular edema secondary to albumin-bound paclitaxel therapy. Arch Ophthalmol 126:1605-1606

21. Ito T, Okuda M (2010) A case of cystic maculopathy during paclitaxel therapy. Nippon Ganka Gakkaishi 114(1):23-27

22. Kajiya S, Hayakawa K, Sawaguchi S (1999) A case of tamoxifen retinopathy. J Eye 16(8):1145-1148

23. Nayfield SG, Gorin MB (1996) Tamoxifen-associated eye disease. A review. J Clin Oncol 14:1018-1026

24. Delval L, Klastersky J (2002) Optic neuropathy in cancer patients. Report of a case possibly related to 5 fluorouracil toxicity and review of literature. J Neurooncol 20:165-169

25. Capri G, Munzone E, Tarenzi E et al (1994) Optic nerve disturbances: a new form of paclitaxel neurotoxicity. J Natl Cancer Inst 86(14):1099-1101

26. Colley SM, Elston JS (2004) Tamoxifen optic neuropathy. Clin Experiment Ophthalmol 32(1):105-106

27. Kanzaki M, Inoue J, Wakakura M et al (2000) Toxic neuro-retinopathy due to tamoxifen. Neuro Ophthalmol Japan 17(3):327-332 